\title{
Design of the Crab label tag with a loop matching feed and a modified dipole structure at $900 \mathrm{MHz}$
}

\author{
Eui-Sun Choi ${ }^{\dagger}$, Hak-Yong Lee $* *$, Jin-Seong Lee***, Kyounghwan Lee ${ }^{* * *}$, \\ Sa-Won Lee****, and Young-Hie Lee*
}

\begin{abstract}
The Crab label tag with a loop matching feed and a modified dipole antenna structure was proposed. The antenna impedance is conjugated easily to a radio frequency identification IC chip impedance by a loop matching feed. The reading range of the crab structure tag is $0.9-1.0 \mathrm{~m}$ from the upper side of the formula milk can lid. The fabricated label tag size is $44.0 \times 44.0 \mathrm{~mm}$. The operating frequency at $-3 \mathrm{~dB}$ return loss is $861.0-929.0 \mathrm{MH}$, and the maximum reading range at the anechoic chamber is $1.5 \mathrm{~m}$.
\end{abstract}

Keywords: RFID, Crab, Label, Tag, Antenna, Milk can

\section{Introduction}

RFID (Radio Frequency IDentification) is a wireless technology for recognizing tag information. Therefore, it requires a tag, a reader, and a reader antenna. The tag consists of an antenna and a semiconductor chip that records information on the semiconductor chip memory and sends it to the reader through the antenna. This information is used to identify an object to which the tag is attached. The tag has a similar function to that of the barcode currently used in a wide range of services. The difference between RFID and the barcode system is that the former uses radio waves instead of light. The RFID system not only works on closed objects as the barcode system does, but it can also recognize tags in remote distances. It can receive information from tags, even through objects, and can recognize a large number of tags at a time. Depending on the power source it uses, RFID is classified into passive or active type. Depending on the frequency, it is classified into 125/135 kHz, $13.56 \mathrm{MHz}, 433$ $\mathrm{MHz}, 900 \mathrm{MHz}$, or $2.4 \mathrm{GHz}$ [1-2]. In addition, depending on the tag type, it is classified into label tag or metal tag [3-5]. RFID technology originated from the equipment developed by Leon Theremin of the former Soviet Union for intelligence warfare. Thereafter, it has been applied and

$\dagger$ Corresponding Author: Department. of Electronic Materials Engineering, Kwangwoon Univerity, Korea. (sunsee@kw.ac.kr)

* Department. of Electronic Materials Engineering, Kwangwoon Univerity, Korea. (yhlee@kw.ac.kr)

** SONOVISION Co., Ltd, Korea. (hyleeantenna@hanmail.net)

*** Department. of Antenna Research, UTRC, Korea. (jslee@utrc.re.kr, khlee@utrc.re.kr)

**** Department of Information Communication and Homenetwork, Chungju College of Korea Polytechnic IV , Korea. (swlee@kopo.ac.kr)

Received: May 25, 2010; Accepted: March 4, 2011 developed diversely as a military technology in automatic answering machines and identification of friend or foe. The 1973 patent by Mario Cardullo used radio wave, sonic wave, and light for communication. It also had a built-in memory for wireless communication. Therefore, it is considered the first true RFID system. In the same year, Steven Deppetal demonstrated RFID technology with limited output for the first time in Los Alamos National Laboratory. Thus far, this is the technology that has been used as the RFID system. Recently, RFID has been used in very diverse applications such as in tracking product history, attaching passports or ID cards to recognize personal information, and recording athletes' data. In addition, it is also used in traffic fare collection systems such as Hi-pass and transit cards, as well as security cards for building access and implantation in human bodies or animals for certain medical purposes. Therefore, RFID is attracting substantial attention as an effective substitute for barcodes. To expand further its application, intensive research and development activities are being undertaken to reduce factory inventory management time, to make physical distribution flow transparent, and to manage the authenticity of expensive commodities. In particular, RFID is now applied to food management in order to prevent food poisoning or death from eating expired or decomposed food. This is achieved by making the management of data such as the expiry date, nutrient facts, and distribution flow efficient and transparent. The current study aims to examine 900MHz-band RFID tags attached to powdered infant formula cans in order to manage effectively powdered infant formula products that are mainly consumed by babies. These are among the many food items which have expiry dates that affect human food safety significantly. 


\section{Design of the tag antenna}

Through the analysis of formula milk cans currently in the market and user environment to which the RFID system would be applied, the tag design specification was determined as shown in Table 1. The formula milk cans have a thin metal plate under their Low Density PolyEthylene (LDPE) lids, which must be taken into consideration for the tag design. As the formula milk cans are cylindrical, the tag should be readable regardless of the direction of its placement.

Table 1. Tag design specification

\begin{tabular}{c|c|c|c}
\hline No. & Items & Spec. & Note \\
\hline 1 & $\begin{array}{c}\text { RFID operating } \\
\text { frequency }\end{array}$ & $908.5-914.0 \mathrm{MHz}$ & Republic of Korea \\
\hline 2 & $\begin{array}{c}\text { Recognition } \\
\text { distance }\end{array}$ & $>1.0 \mathrm{~m}$ & Directionless \\
\hline 3 & RFID chip & Impinj's Monza 2 & $53-\mathrm{j} 160 \Omega @ 910 \mathrm{MHz}$ \\
\hline 4 & Tag size & $44.0 \times 44.0 \mathrm{~mm}^{2}$ & Inlay(t=0.068 mm $)$ \\
\hline 5 & $\begin{array}{c}\text { Attachment } \\
\text { location }\end{array}$ & $\begin{array}{c}\text { On the formula milk } \\
\text { can }\end{array}$ & $\begin{array}{c}\text { Upper side of } \\
\text { LPDE }\end{array}$ \\
\hline 6 & Reader & $\begin{array}{c}\text { Alien Co. } \\
\text { ALR-9800 }\end{array}$ & $\begin{array}{c}30 \mathrm{dBm}(\mathrm{Max}) \\
\text { Republic of Korea }\end{array}$ \\
\hline 7 & Reader Antenna & $\begin{array}{c}\text { Alien Co. } \\
\text { ALR-9611-CR }\end{array}$ & 6dBic(Max), RHCP \\
\hline
\end{tabular}

To satisfy the specifications presented in Table 1, a simulation using MWS's CST 2008 was carried out. The impedance of the tag antenna was conjugated to that of Impinj's Monza 2 RFID chip when the tag antenna is attached to the formula milk can lid. Fig. 1 shows the structure of the tag antenna designed for a formula milk can. For the tag to fit the sticker size attached to the formula milk can lid and be recognized at a distance of 1.0 $\mathrm{m}$ or more in any direction vertical to the reader antenna, the dipole antenna part was deformed into "crab" leg shape. For the impedance of the tag antenna to be conjugated to that of Impinj's Monza 2 RFID chip, a "loop-shaped" matching terminal was used. The proposed tag allows adjustments of the width (Fig. 1(c)) and height (Fig. 1(f))

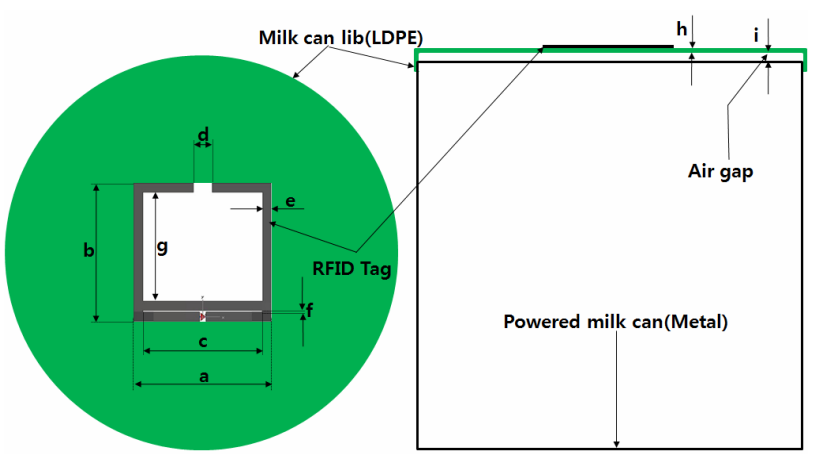

Fig. 1. Tag antenna structure for the powdered milk can of the loop matching terminal, facilitating easier control of the inductance and capacitance. This in turn results in the conjugation of the RFID chip and tag antenna. For the current study, the tag for the powered baby formula can is named "Crab Tag" as its antenna shape is similar to that of a "crab."

To investigate the possibility of filter application, the triplexer was designed, simulated, and manufactured using the Advanced Design System and HFSS which is used in electro-magnetic analysis. The frequency response characteristics of the manufactured triplexer were measured by a vector network analyzer.

$$
\text { Return } \operatorname{loss}[\mathrm{dB}]=20 \log _{10}\left|\frac{z_{a}-z_{b}}{z_{a}+z_{b}}\right| \quad<\text { Formula 1> }
$$

$z_{a}:$ Impedance of the tag antenna

$z_{b}:$ Impedance of the RFIC Chip

The simulated tag antenna is attached to the formula milk can. Fig. 2 shows the graph of the changes in resistance and reactance of impedance depending on the size of "f," whereas Fig. 3 shows the graph for the return loss of each impedance. In Fig. 2, as the value of "f" increases, the resistance decreases, and the reactance

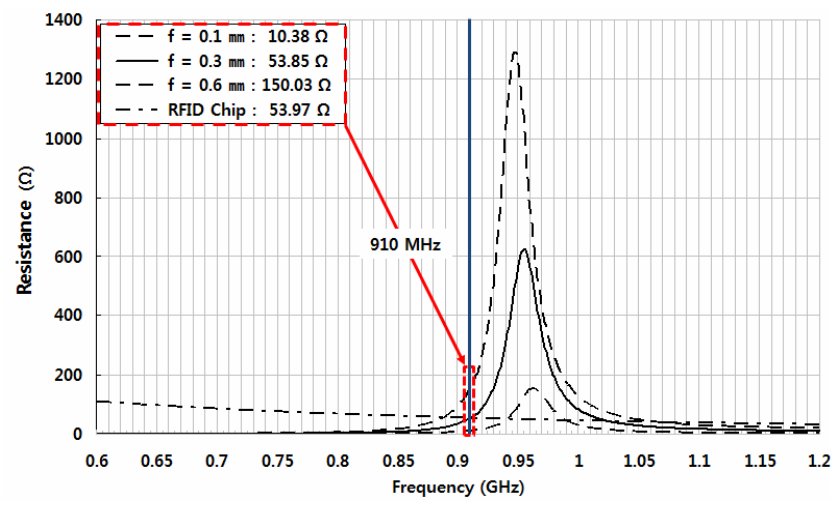

(a) Resistance

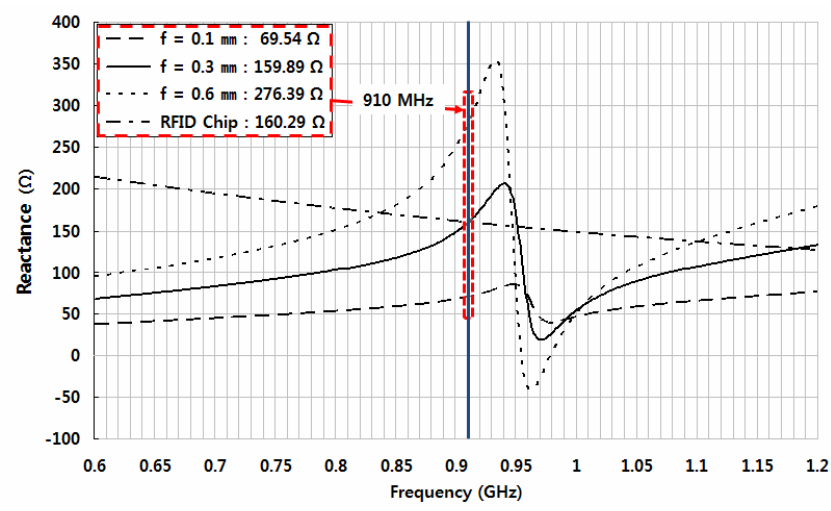

(b) Reactance

Fig. 2. Impedance values of the tag antenna (simulation) 
increases. The range of increase or decrease appears to be bigger in the reactance than in the resistance. The chip impedance is calculated to use the Monza 2 datasheet of Impinj Co [6]. Fig. 3 shows the reflection factors calculated using Formula 1 with reference to chip impedance. When "f" is $0.3 \mathrm{~mm}$, the reflection factor becomes the smallest at $910 \mathrm{MHz}$ as $-48.30 \mathrm{~dB}$, at which -3 $\mathrm{dB}$ bandwidth is $67 \mathrm{MHz}$ (872-939 $\mathrm{MHz})$. The tag size optimized as a result of the simulation of the RFID system for the powered baby formula can tag is " $\mathrm{a}=44 \mathrm{~mm}, "$ " $\mathrm{b}=44$ $\mathrm{mm}, "$ "c=38 mm," "d=6.0 mm," "e=3 mm," "f=0.3 mm," "g=37.97 mm," "h=0.77 mm," and "i=1.3 mm."

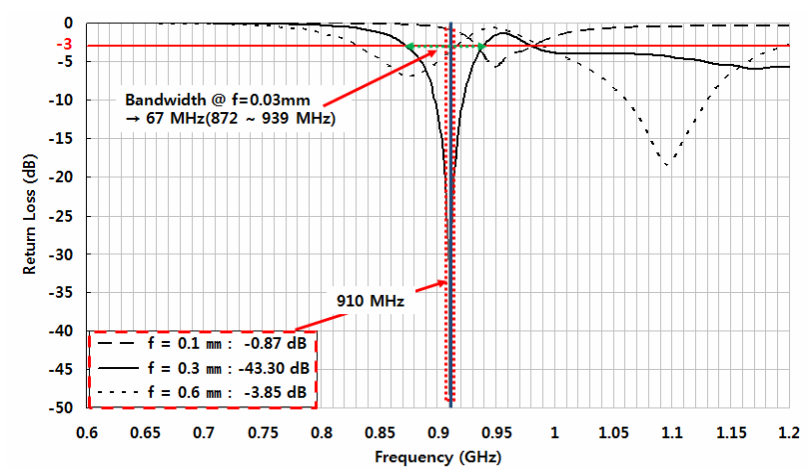

Fig. 3. Return loss values of the tag antenna (simulation)

\section{Fabricated and measured the tag}

Fig. 4 shows a tag fabricated by forming a copper pattern with a thickness of $0.018 \mathrm{~mm}$ on a $0.05 \mathrm{~mm}$-thick Polyethylene Terephthalate with a relative permittivity of 3.3. Through measurements and experiments, the tag antenna size was optimized as shown in Fig. 4, which is "a=44 mm," "b=44 mm," "c=26 mm," "d=6 mm," "e=3 mm," and $\mathrm{f}=0.3 \mathrm{~mm} . "$

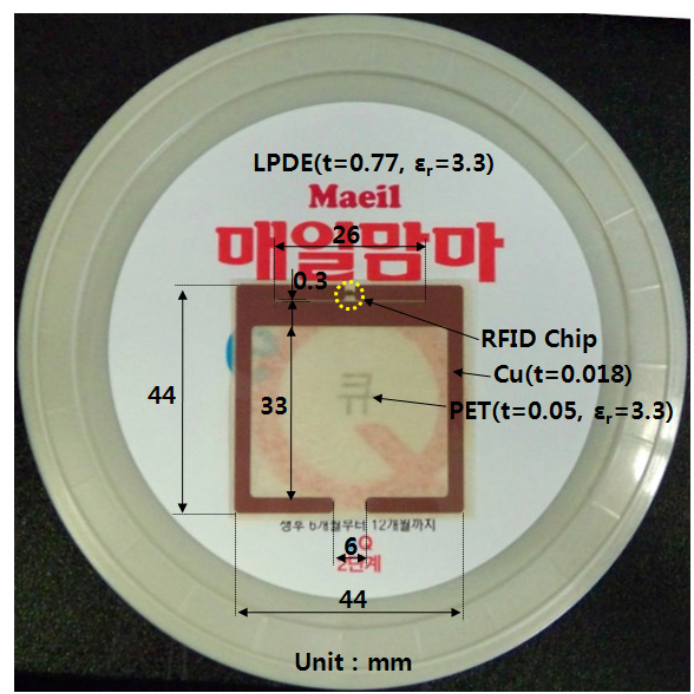

Fig. 4. Fabricated crab tag

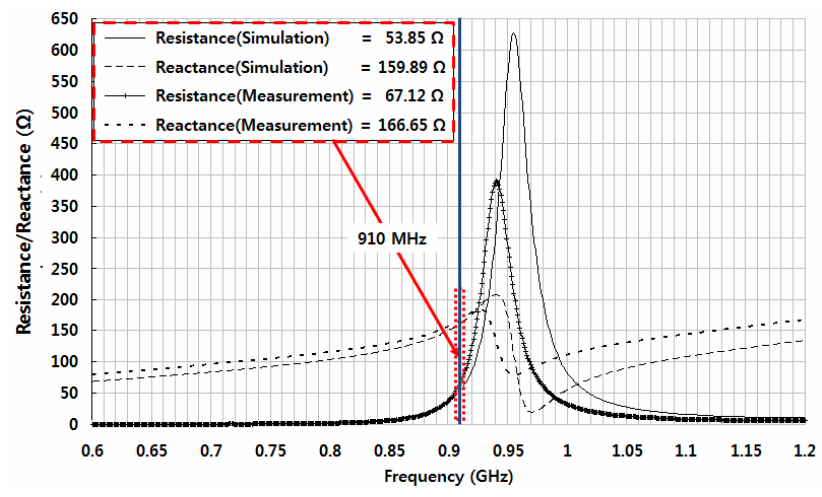

Fig. 5. Comparison of the tag antenna impedance results (measurement and simulation)

Fig. 5 shows the graph comparing the tag impedance measured from the crab tag attached to the baby formula milk can lid with the simulation results. When the tag was attached to the formula milk can lid, the resistance of the tag impedance decreased, and its reactance increased. This implied that the impedance changed depending on the medium to which the tag was attached and the metal plate of the formula milk can. When the tag antenna was attached to the formula milk can lid and when its impedance which the test system used in reference [7] was measured at $910 \mathrm{MHz}$, the impedance was $67.12 \Omega$ at the resistance and $166.65 \Omega$ at the reactance parts, respectively. If the impedance of the RFID chip is 53.97-j160.29 $\Omega$, there might be some error in measuring the tag antenna impedance. Fig. 6 shows a graph comparing the results of simulation and the measurement of return loss. The change in impedance resulted in a change in return loss. In the simulation, the return loss was $-48.30 \mathrm{~dB}$ at $910 \mathrm{MHz}$ but was reduced to $-18.71 \mathrm{~dB}$ at the time of the benchmark test (BMT). During this period, the time frequency also changed to $901 \mathrm{MHz}$. The reflection factor was moved to a lower frequency because the tag was attached to the formula can. Based on $-3 \mathrm{~dB}$ return loss, the operating range is $861-929 \mathrm{MHz}$, and the bandwidth is about $68 \mathrm{MHz}$, which includes the entire RFID operating frequency range in Korea.

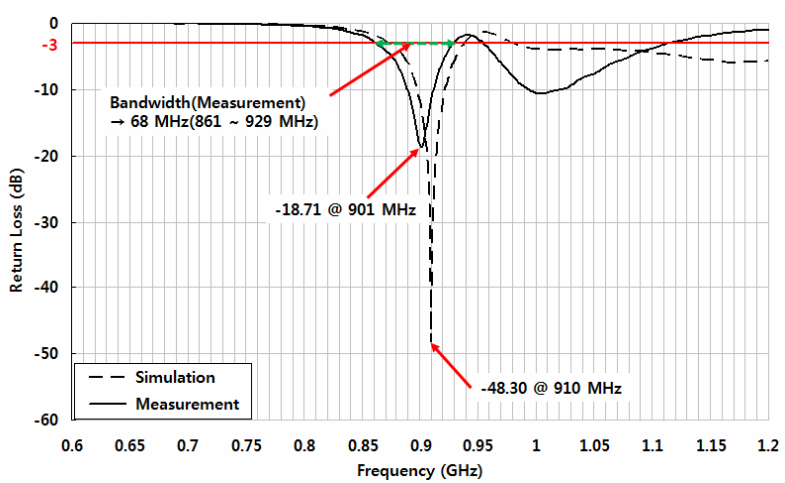

Fig. 6. Return loss of a crab tag (measurement and the simulation) 
To ensure the more accurate measurement of the crab tag, its sensitivity and recognition distance were measured at the electromagnetic anechoic chamber in "Songdo RFID/USN Center in Incheon (Korea)." Sensitivity is the minimum power of the RFID reader when the tag is operated at each frequency. This means that the impedance of the tag antenna matches well that of the RFID IC chip as the sensitivity value is lower. Reference 8 describes the sensitivity measurement method. Fig. 7 shows the sensitivity measurement system that consists of a TESCOM TC-2600A RFID tester, a G94007A RFID highpower amplifier, and an LG Industrial Systems' reader antenna (Max gain : $6 \mathrm{dBic}$ ). With a distance of $1 \mathrm{~m}$ between the tag and Tx antenna and the crab tag attached to the formula milk can, the tag sensitivity was measured. Fig. 8 shows the recognition distance measurement system that consists of Alien's reader ALR-9800 (KOR) and a circular polarization (RHCP) reader antenna; the crab tag was attached to the formula can. The maximum recognition range is $10 \mathrm{~m}$. The sensitivity result is shown in Fig. 9. Tag sensitivity was measured by the unit of $10 \mathrm{MHz}$ at the range of 860-960 MHz. It appeared to be higher as the relative measurement value was lower. According to the measurement results, the sensitivity of the tag was $2.1 \mathrm{dBm}$ at $910 \mathrm{MHz}$. A comparison of these results with the characteristics in Fig. 9 suggests that the tag worked properly. The performance seemed to result from the slight change in impedance due to RFID chip bonding. The maximum recognition distance of the crab tag measured with the recognition distance measurement system shown in Fig. 10 was $1.5 \mathrm{~m}$ for all sample tags.

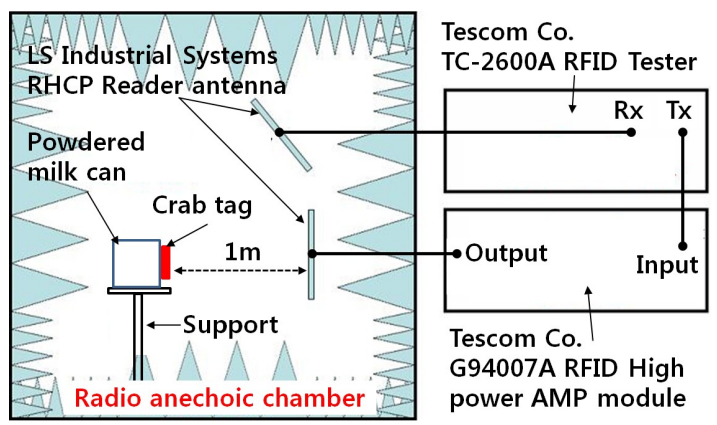

Fig. 7. Sensitivity measurement system

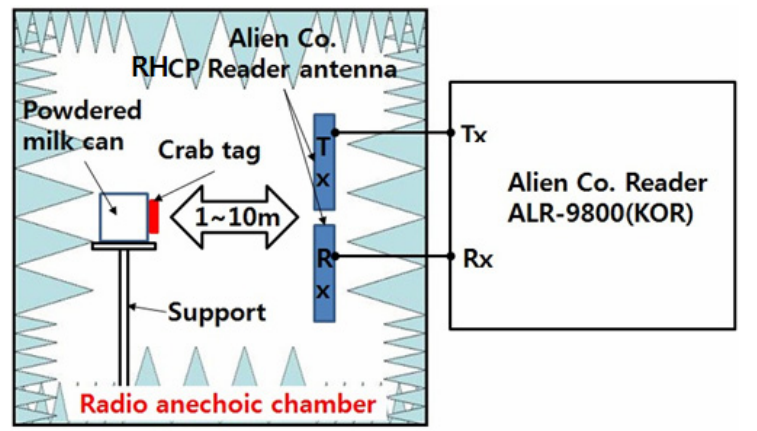

Fig. 8. Reading range measurement system

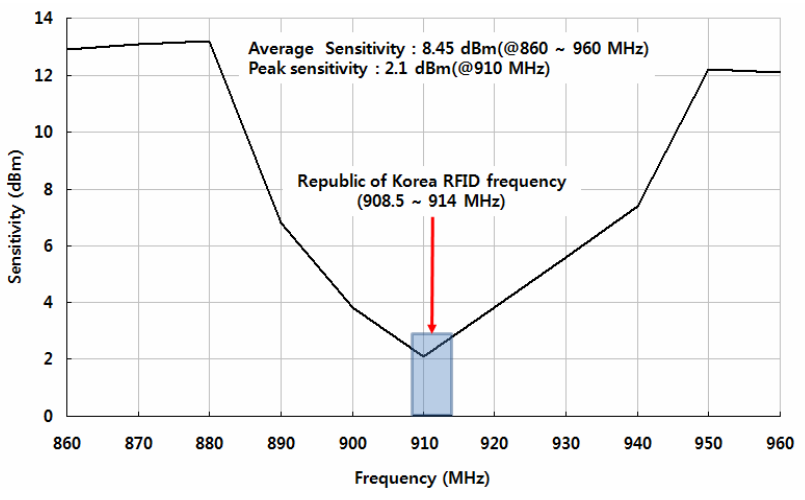

Fig. 9. Sensitivity and max reading range of the crab tag

However, in BMT, the tag should be placed more parallel to the ground than the maximum recognition distance, and it should be attached to cylindrical formula milk cans. Therefore, the tag should be recognizable at any same distance regardless of its tag orientation. For this reason, the measurement system was configured as shown in Fig. 10, and the formula milk can to which the tag was attached was rotated $90^{\circ}$ clockwise for measurement. As the result of the measurement of 50 tags, a uniform distance of 0.9-1.0 $\mathrm{m}$ was obtained.

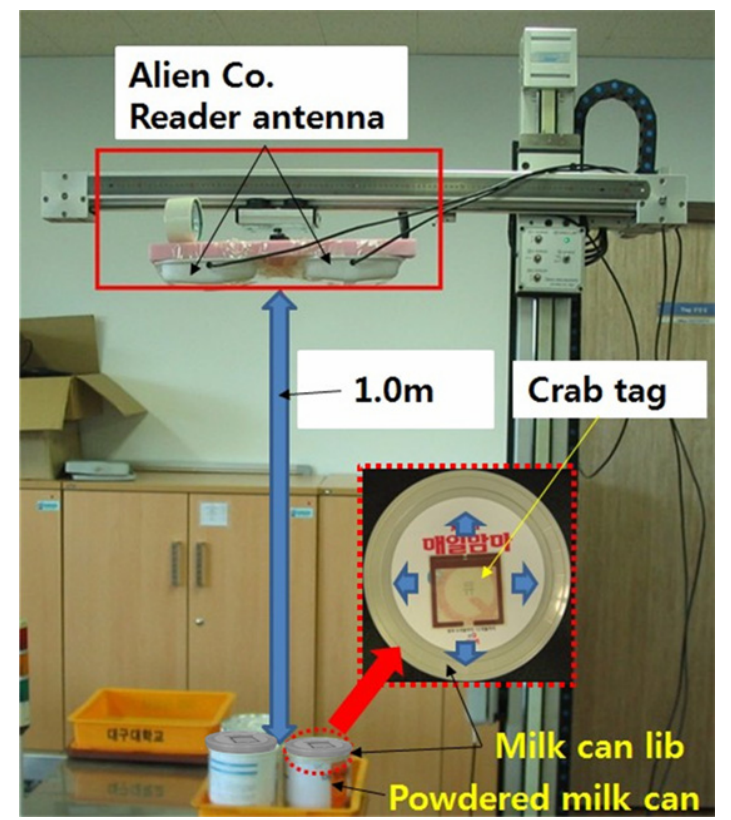

Fig. 10. Tag reading range measurement system showing the angle of the reader antenna

\section{Conclusion}

The current study proposed the "crab tag" using a modified dipole antenna structure that includes a loopshaped matching terminal. This terminal facilitates an impedance air-interface between the RFID chip and antenna. Also, the dipole antenna structure has a "crab" 
shape that provides the following structural advantage. Although the tag is label type whose size is $44 \times 44 \mathrm{~mm}^{2}$, and the tagged surface and the metal rim are about $2.0 \mathrm{~mm}$ apart from each other, the crab tag has a relatively long recognition distance of $0.9-1.0 \mathrm{~m}$ when attached parallel to the ground surface. When measured in a radio-wave nonreflecting chamber, the maximum recognition distance is about $1.5 \mathrm{~m}$, and under the actual operating environment, it is sometimes measured as $2.0 \mathrm{~m}$ or longer. This can be due to the increase in recognition distance as the reflection of the radio wave increases. The result of measurement in the radio anechoic chamber confirms that the crab tag matches the best at Korean RFID frequencies. The proposed crab tag with these characteristics facilitates antenna shape change and impedance air-interface. Due to its small size, it is also suitable for applications that need to be recognized within the distance of $1.0 \mathrm{~m}$. It is currently used for formula milk cans in Korea in order to promote transparency in distribution and food safety management.

\section{Acknowledgement}

This work was supported in part by MKE \& NIPA (Ubiquitous Technology Research Center)

\section{References}

[1] K. Finkenzeller, RFID Handbook, 2nd edition, John Wiley \& Sons, England, 2003.

[2] G. De Vita and G. Iannaccone, "Design criteria for the RF section of UHF and microwave passive RFID transponders, Microwave Theory and Techniques," IEEE Trans. Vol. 53. Issue 9, pp. 2978-2990, Sep. 2005.

[3] Q, Chen and B. Hu, "Novel UHF RFID tag antenna with shorted stubs mountable on the metallic objects," ICMMT 2008. International Conference, Vol. 4, pp. 1822-1824, Apr. 2008.

[4] D. Kim and J. Yeo, "Low-Profile RFID Tag Antenna Using Compact AMC Substrate for Metallic Objects," IEEE AWPL, Page(s):1 - 3, 2008.

[5] K.H. Lee and Y.C. Chung, "High gain Yagi-Uda UHF RFID tag antennas," IEEE AP-S International Symposium, pp.1753-1756, Jun. 2007.

[6] NXP Semiconductors Co., "SL3ICS1002 G2XM," Rev. 3.2, Jul. 2008.

[7] J.S. Jung, J.S. Lee, K. Lee, J.M. Yang, "900 MHz Common RFID Label Tag Impedance Analysis Using Produced Prove Station," 2010 Winter Symposium, Korean Information and Communications Society, Vol. 41, pp.165, Feb. 2010.

[8] http:/www.epcglobalinc.org/, "Static Test Method," Rev 1.9.4, May 2008

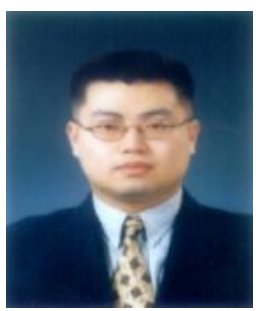

Eui-Sun Choi He received his M.S. and Ph.D. degrees in Electronic Materials Engineering from Kwangwoon University. His research interests are microwave dielectric ceramics, LTCC, antenna, and RFID.

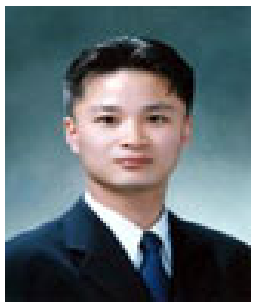

Hak-Yong Lee He received his M.S. and and Ph.D. degrees in Electric Wave Engineering from Kwangwoon University. $\mathrm{He}$ is the $\mathrm{CEO}$ of SONOVISION Co., LTD. His research interests are sensor, antenna, and RFID.

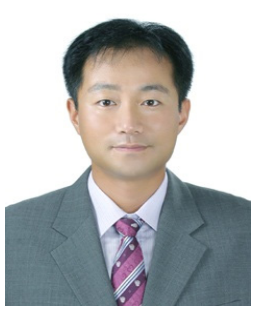

Jin-Seong Lee He received his M.S. and $\mathrm{Ph} . \mathrm{D}$. degrees in Electric Wave Engineering from Kwangwoon University. He is a researcher of the Ubiquitous Technology Research Center, Korea. His research interests are antenna and RFID.

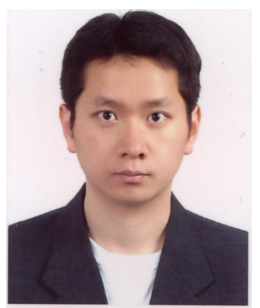

Kyounghwan Lee He received his B.S and M.S. degrees in Computer and Communication Engineering from Daegu University. He is a researcher of the Ubiquitous Technology Research Center, Korea. His research interests are antenna and RFID.

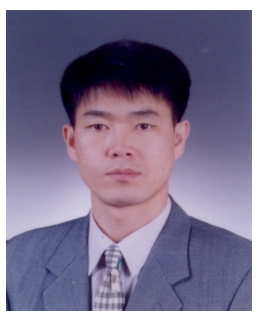

Sa-Won Lee He received his B.S. and M.S. degrees in Electronic Engineering from Cheongju University. $\mathrm{He}$ is a professor of the Department of Information Communication and Homenetwork at Chungju College of Korea Polytechnic IV. His research interests are filter, antenna, and RFID.

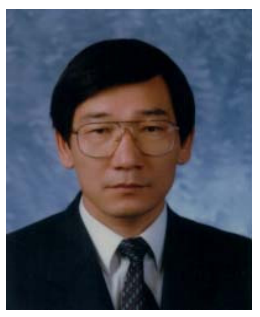

Young-Hie Lee He received his M.S. and Ph.D. degrees in Electrical Engineering from Yonsei University. He is a professor of the Department of Electronic Materials Engineering at Kwangwoon University in Seoul, Korea. His research interests are solgel processing, piezoelectric ceramics, thin film, microwave dielectric ceramics, sensor, and memory. 